\title{
NATURE AND MUSIC: A GENERAL METHOD FOR DESIGNING A COMPOSITION FROM AN IMAGE
}

\author{
G. DONATI ${ }^{1} \&$ P.P. STRONA ${ }^{2}$ \\ 1"Conservatorio Giuseppe Verdi", Torino, Italy. \\ ${ }^{2}$ Independent Researcher, Torino, Italy.
}

\begin{abstract}
The aim of this paper is to provide a general method to enable a composer to base his musical works on images. A kind of connection is established between the world of sounds and pictures, taking into account mainly subjects taken from nature. First of all the main relationships between nature, photography and music are considered, focusing, in particular, on common geometrical aspects. Then the proposed method is explained in detail, high-lighting the general validity of the approach and how it is consistent with the main aspects of the musical discourse. The composer's freedom and creativity are also considered. Finally, the method is illustrated by examples of musical compositions based on a landscape photograph taken in the "Gran Paradiso National Park', in the Italian Alps.
\end{abstract}

Keywords: chords, composition, geometry, Goldberg variations analysis, images structures, Joplin ragtimes, landscapes, musical lines, musical signals, musical structures.

\section{INTRODUCTION}

In nature everything grows out of a process of combining small elements to create larger ones, from atoms to the whole universe. In his conceptual and real constructions man has always imitated this way of organizing matter.

With respect to theoretical and abstract matters, using mathematics and geometry, man developed very complex models to investigate and understand natural phenomena: the aim was not only knowledge and technological application but also the investigation of artistic issues too. The heart of the processes that enable man to conceive and build very large and complex theoretical systems consists in selecting the basic elements and defining the rules to be followed to connect them with each other. Finite and Boundary Elements numerical methods (FEM, BEM), which can produce models of almost everything in the world around us and simulate its behavior, are a quite recent and significant example of this.

As far as real constructions are concerned, everything that man has designed and built in the course of history, from the beginnings until now, can be taken as examples. Small and great works have been built using the same method, putting small, simple elements (stones, masonry, beams, etc.) together to make other bigger and more complex structures, from Stonehenge to the Egyptian Pyramids, from the great cathedrals to skyscrapers, suspension bridges and the ever-bolder constructions of modern times.

If we consider the languages man has developed to communicate sounds and images, we find they are often more powerful and effective in communicating emotions and concepts than words are.

A photographer designs his images and a musician designs his compositions using a method very close to that of the architect: as in nature, they combine simple and smaller elements to make other bigger ones, and geometry plays a basic role in this procedure.

In the case of landscape photography, the elements already exist in the scenery in a single moment and place: the photographer utilizes them to create a unique meaningful and artistic image. The geometrical features are essential, first of all in defining the frame, then in considering the harmony of lines and colors. 
In the case of music, its fundamental physical, mathematical and geometrical aspects have to be considered: physical in the essence of its sounds and vibrations, mathematical as a non-linear function, as a signal, $\mathrm{f}$ (time), and geometrical with reference to the procedures of assembling small ideas to create a bigger and more complex discourse. The musician plays with the sounds, changing their basic parameters, generating melodies, rhythms, and timbres.

These aspects are essential in every musical composition man has made, but they are particularly evident in J.S. Bach's [1-4] compositions: he was a musician and a scientist at the same time, contributing probably more than anyone else to define and generalize the tonal system, a method of writing music everywhere applied in western countries from the end of the 17th to the end of the 19th century, and in part still today.

Bach wrote his music as a great architect designs his buildings: he created a few small simple elements and arranged them following a few simple rules, mainly geometrical, to write wonderful, magnificent music.

An analysis of any Bach composition will lead to a better understanding of his way of writing music. The 'Goldberg Variations BWV988' [5] for two harpsichord keyboards, one of the most important and greatest, and particularly the variation n. 8 (Fig. 1), where these geometrical aspects are so evident, is an excellent example.

Two main musical ideas are presented in the first bar (Fig. 2a, b) respectively on the right hand (upper stave) and on the left (lower stave). Bach also generates corresponding mirror-like structures (Fig. 2c, d). Now if the whole variation is considered, we realize that it has been constructed by combining the above four ideas in Fig. $2 \mathrm{a}-\mathrm{d}$ in every possible way, according the combinations synthesized in Fig. 3. Nothing else!

It will easily be seen that written music (mainly melody and rhythm) can always be shown as an $\mathrm{f}(\mathrm{x})$ which describes its note lines: this approach can be used to analyze the composition better, as above, but new intuitions and ideas in an interdisciplinary perspective could also arise from that.
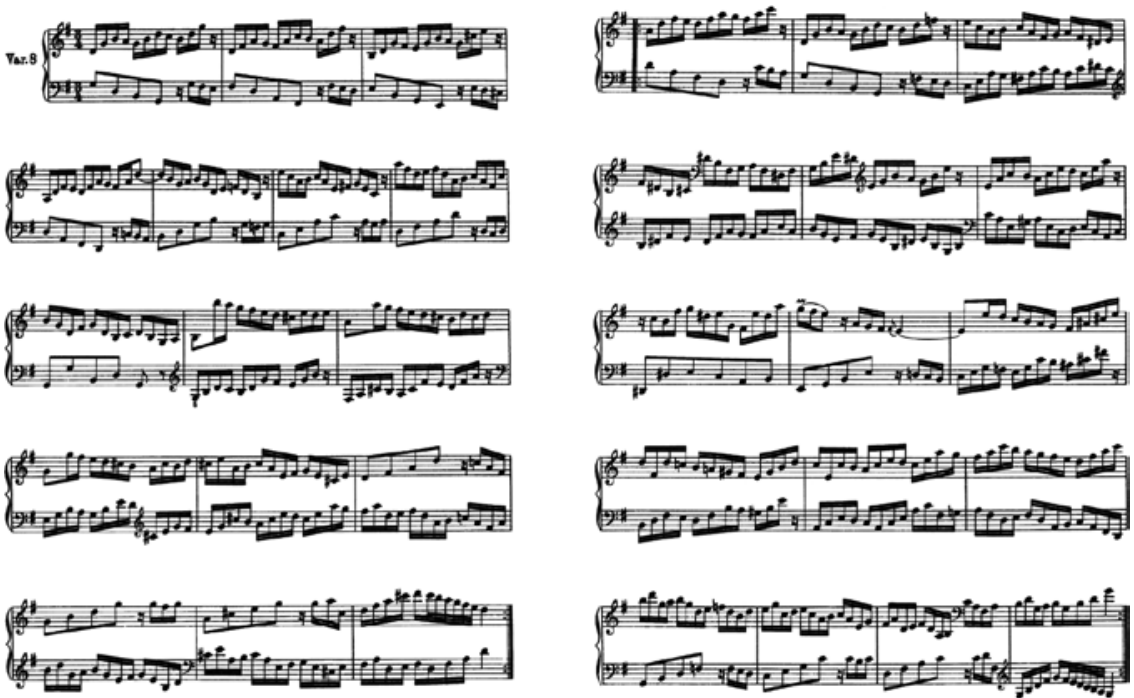

Figure 1: Goldberg Variation n. 8. 


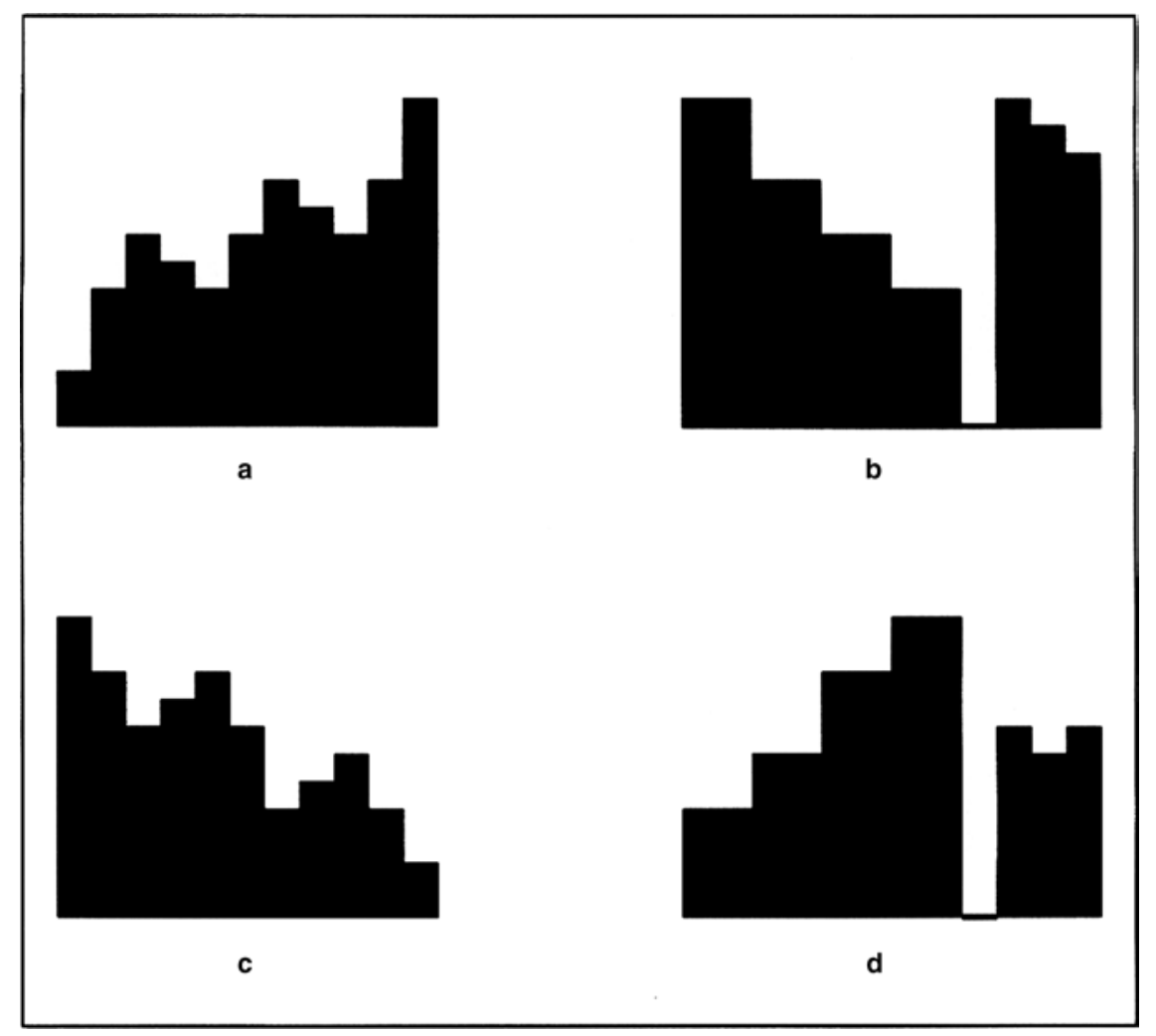

Figure 2: Goldberg Variation n.8, basic musical ideas.

For example, if the skyline notes of other Bach compositions were designed as before, their indentation would appear very clear and characteristic: in fact Bach moves his ideas in sound space changing direction continuously, searching for newer and newer energy points to sustain his discourse and develop it. In this respect, Bach's music is very similar to jazz and particularly to bebop.

As we observe nature we notice frequent indented lines, small and big, for example, in mountain shapes, in sea and lake coast lines, in stones, leaves and flowers and so on. The same is true for man-made buildings and city skylines.

Perhaps that was why Scott Joplin, the greatest Ragtime composer, used tree leaves and flowers names as titles of several of his compositions (Maple leaf, Roseleaf, etc.). Ragtime [6] is very important in the history of music, significantly to the development of jazz: so you can go from Bach to jazz by way of leaves, flowers and the Joplin ragtimes. What is most important is: 1) geometry in nature and music, 2) the assembling and combining techniques, 3) the architectural creation of conceptual, real and musical structures.

Figure $4 \mathrm{a}-\mathrm{d}$, represents some Joplin ragtime themes as skylines. With a little effort of the imagination they might represent cities, industrial buildings or landscapes or other subjects in skylines to be observed in nature.

But the above journey from music to nature could be made in the opposite direction, from nature to music: for example, a composition could be written starting from a landscape. A suitable set of rules would be needed to do that. But, to link subjects in nature and music, another step is 


\begin{tabular}{|c|c|c|c|}
\hline \multicolumn{2}{|c|}{ Bars } & \multicolumn{2}{c|}{ Variation $\mathrm{n} .8$} \\
\hline From & To & Left Hand & Right Hand \\
\hline 1 & 4 & b & a \\
5 & 8 & d & c \\
9 & 12 & a & b var. \\
13 & 16 & c & d \\
\hline 17 & 18 & b & a \\
19 & 20 & a & c \\
21 & 22 & C & A \\
23 & 24 & d var. & a var. \\
25 & 26 & a & b var. \\
27 & 28 & a-c & a var. \\
29 & 32 & d & c \\
\hline
\end{tabular}

Figure 3: Var. n.8, combinations.

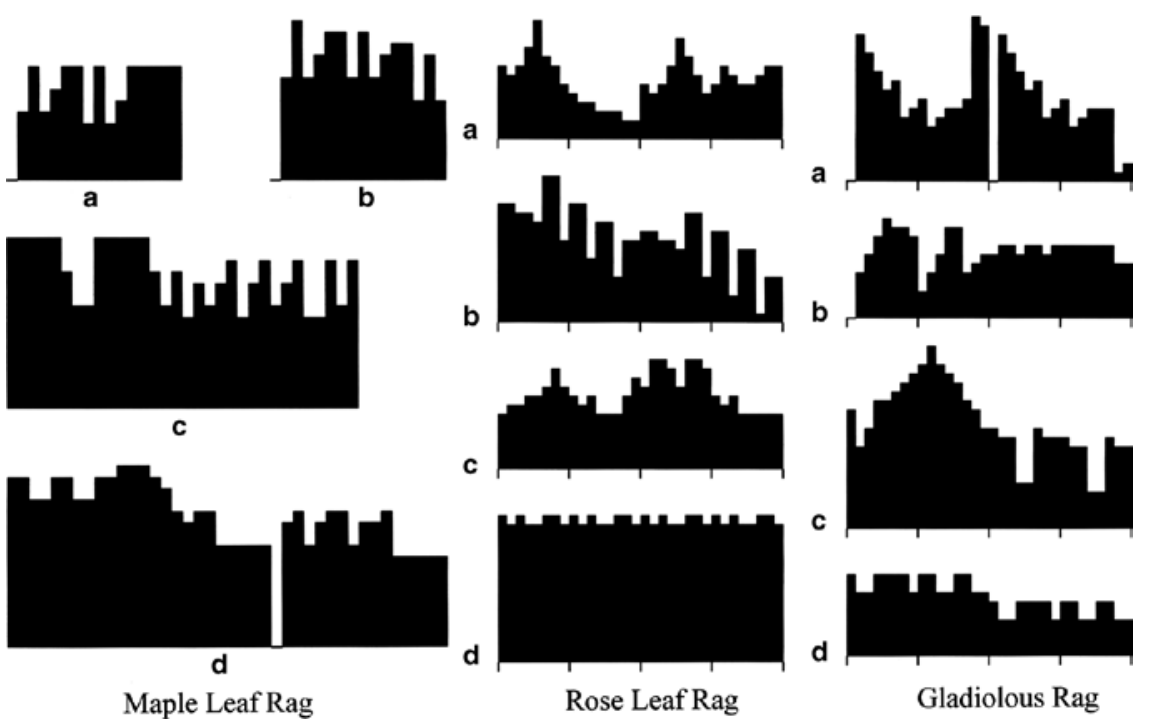

Figure 4: Scott Joplin Ragtimes. 
required: the creation of an image of the subject, using a photograph, or else a drawing or a painting. The subjects taken from nature must be filtered by the eyes and the mind of the observer, reflecting his emotions. Hereafter, only photography will be considered.

\section{THE METHOD}

The method proposed is based on the following main criteria:

- The method will be applied to the functions which describe the lines defining the geometrical shapes in an image.

- The method must be suitable to take into account the structure of the musical language and its basic elements: melody (horizontal dimension), harmony (vertical dimension), rhythm, timbre.

- The method must include rigorous rules, consistent with each other and with the features of the corresponding musical language elements, but it must also leave the composer free in designing his music.

First of all the subject, for example, a landscape to be interpreted by a musical composition must be selected. But the first step will be to interpret it graphically, taking a photograph. In this connection the most important choices to be made are:

- the point of observation.

- the moment, the quality of the light.

- the lens, that is the frame and the geometrical deformation of the subject.

- eventually a further graphical deformation of the image taken, if in this way some perception would be better focused.

By these choices the photographer is able to interpret the subject and how it is perceived, revealing his feeling, his emotions and influencing the subsequent musical interpretation.

Generally, according to the shapes of the images, one or more lines, and the corresponding $\mathrm{f}(\mathrm{x})$, able to describe it, can be defined. For example, in Fig. 5 a photograph of an Alpine landscape is shown (Alpi Graie, Italian side): here there is an image of mountains and valleys and several related lines are needed to describe the scenery well.

To explain the method, the case of only one $\mathrm{f}(\mathrm{x})$ will be considered. Now the problem is: how to interpret the image musically?

As far as the generation of musically consistent materials is concerned, the first thing that comes to mind might be to associate the notes with the corresponding $f(x) y$-axis values. But this is a very
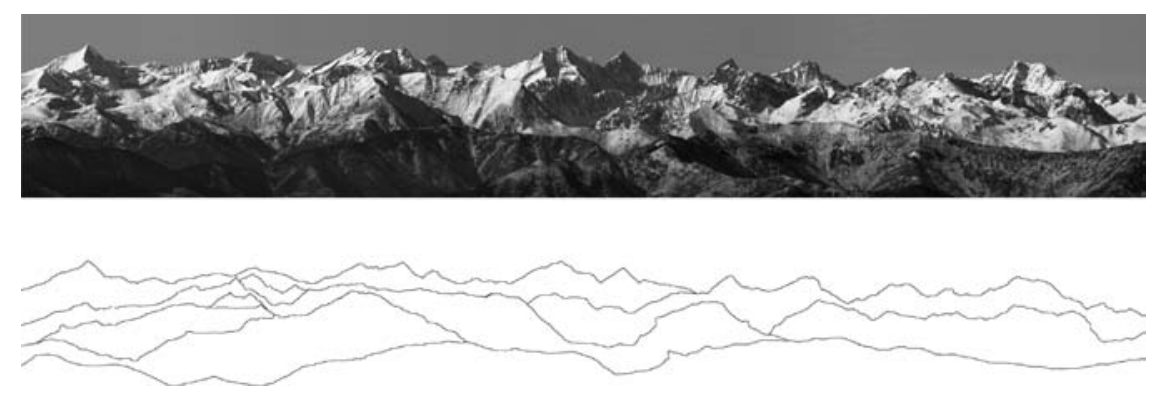

Figure 5: ‘Alpi Graie’ landscape. 
uninteresting idea: it would mean translating quite automatically the $\mathrm{f}(\mathrm{x})$ into a melody, without any evaluation of the other aspects of the musical discourse. The composer would have almost no degree of freedom in composing his music.

Therefore a basic 2D element, not the single notes, has been defined, to be consistent with one of the main features of musical language, the two dimensions, horizontal and vertical, melody and chord. This element has been divided into four equal parts, four quadrants. The composer can associate quite freely to the quadrants four notes which could be used both as notes in a melody or notes in a chord.

The composer also has to decide, again quite freely, the numerical sequence of the quadrants, according to one of the existing permutations: in his criteria in this decision he would identify the most interesting sequence considering the path and features of $f(x)$, on which to base a beautiful and interesting composition. For example, he can decide to make the numbers and the notes increase as $\mathrm{f}(\mathrm{x})$ increases and vice versa. The four basic notes can also be selected by the composer quite freely, for example, at diatonic or chromatic intervals, or at longer and more varied intervals.

In Fig. 6a and $\mathrm{b}$ a possible number and sequence of notes for the basic element is shown: these have been utilized in the application example shown later.

If the quadrant notes have to be considered as chord notes, the voices must be inserted in the basic element too: therefore each element has been divided into four equal zones corresponding respectively to the main voices, Bass, Tenor, Contralto and Soprano. Therefore, the basic element has four quadrants and sixteen zones.

The sequence numbers of the zones can also be selected quite freely by the composer as the sequence of quadrant numbers.

In Fig. 7, the basic element is shown together with a possible quadrant and zone arrangement: it has been utilized in the application illustrated below.

This basic elements structure could be also applied to take into account the other aspects of the musical language, particularly rhythm and timbre: for example, the bars divisions could be inserted in the quadrants or a selection of instruments used instead of notes.

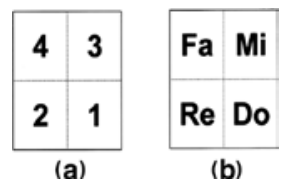

Figure 6: Quadrants and notes sequences.

\begin{tabular}{|c|c|c|c|}
\hline$v 4$ & $v 3$ & $v 4$ & $v 3$ \\
\hline$v 2$ & $v 1$ & $v 2$ & $v 1$ \\
\hline$v 4$ & $v 3$ & $v 4$ & $v 3$ \\
\hline$v 2$ & $v 1$ & $v 2$ & $v 1$ \\
\hline
\end{tabular}

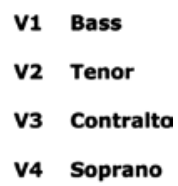

Figure 7: Basic Element. 
The main problem is to define the dimensions and the number of the basic elements, in order to consider the whole $\mathrm{f}(\mathrm{x})$. The following main considerations can be made:

- Defining the dimensions of the basic elements means to define the degree of approximation of $f(x)$, since the $f(x)$ is a continuous function and it is discretized by the basic elements.

- Generally speaking it would be possible to utilize not only the basic square elements, but also the rectangular ones, that is to define different scales for the $\mathrm{x}$-axis and the $\mathrm{y}$-axis.

- The dimensions and the number of elements define:

a) regarding the $y$-axis, the range of the notes used in the composition.

b) regarding the $\mathrm{x}$-axis, the total time of the composition.

Now the composer can begin to create his music according to the $f(x)$ in the following main ways:

- By creating a melody with the notes that $\mathrm{f}(\mathrm{x})$ meets in the related quadrants of the basic elements.

- By creating a sequence of chords taking into account not only the notes in the quadrants but also the voices in the zones crossed by $\mathrm{f}(\mathrm{x})$. The chords are defined by a few strict rules, but the composer is free to select the type of chords (major, minor, seventh, etc.) and the arrangement of notes. So he can create a musical composition which is interesting and beautiful or dull and scholastic according to his creative and imaginative powers.

At this point the composer could conclude his composition. Otherwise he could continue to develop it in several different ways, for example he could:

- Utilize the above melodies and chords as a basis from which to develop a longer and more complex composition.

- Repeat the above procedure changing the number and the shape of the basic elements, the arrangement of numbers and the notes in the quadrants, as well as the arrangement of the voices in the zones. In this way several new melodies and chord sequences could be created, each of them based on the same $f(x)$, rather like variations on a theme, the theme being in this case is not a musical idea but an image.

\section{AN APPLICATION OF THE METHOD}

The photo presented in Fig. 8 shows a partial view of mountains in the 'Gran Paradiso' group (4061 m), in Italian Alps, in its southern side, at sunset.

Giosué Carducci (1835-1907), a famous Italian poet, often traveled in this region: in a famous poem he described these mountains as 'Dentate scintillanti vette' (Jagged sparkling summits).

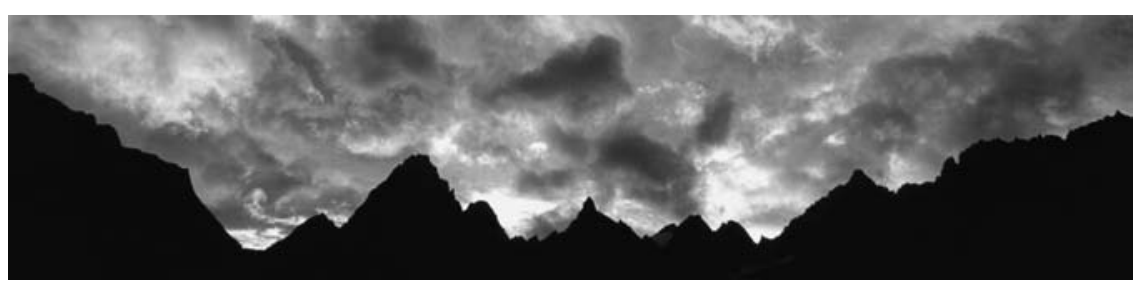

Figure 8: Gran Paradiso, Becco Meridionale della Tribolazione. 
The photo was taken from the 'Rifugio Pontese' $(2217 \mathrm{~m})$. The main mountain shown here is one of the most important in the Alps, both for its beauty and its climbing history: the 'Becco Meridionale della Tribolazione' (3360 m). The photo was taken by P.P. Strona in September 1997, on the evening before climbing it.

The skyline has been traced through some hundred points and has been interpolated to define a function $f(x)$. The $x$ scale has been changed a little to better express the jagged effect of the outline. The line generated is shown in Fig. 9.

To define the dimensions of the basic element, it was decided to have only a few sounds and that the composition should last only a few minutes. So the seven square elements shown in Fig. 10, on the left of the y-axis, were considered; each basic element including four quadrants.

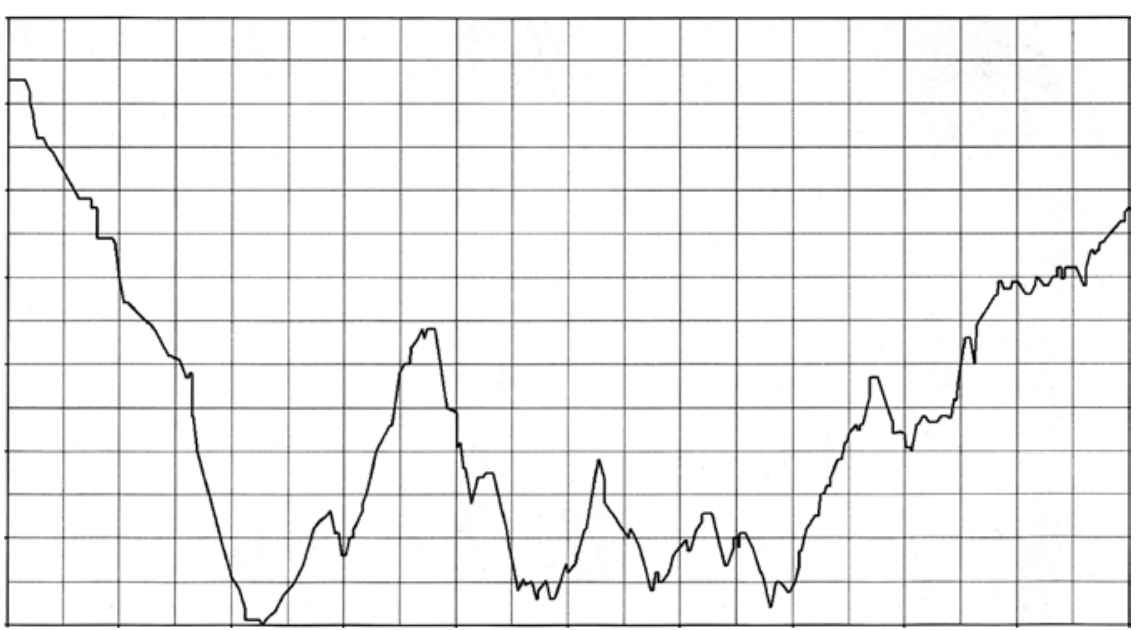

Figure 9: Gran Paradiso, Becco Meridionale della Tribolazione: Skyline f(x).

\begin{tabular}{|c|c|c|c|c|c|c|c|c|c|c|c|c|c|c|c|c|c|c|c|c|c|}
\hline Mi & $R_{\theta}$ & M & Re & Mi & Re & Mi & Re & M & Re & Mi & Re & $\mathrm{M}$ & Re & Mi & Re & M & Re & Mi & Re & M & Re \\
\hline$D_{0}$ & $\mathrm{si}$ & $7^{D_{0}}$ & si & $D_{0}$ & $\mathrm{si}$ & $D_{0}$ & Si & $D_{0}$ & $\mathbf{S i}$ & $D_{0}$ & Si & Do & $\mathrm{si}$ & Do & si & $D_{0}$ & $\mathbf{s}$ & $D_{0}$ & Si & Do & $\mathbf{S i}$ \\
\hline$R_{e}$ & Do & to & $\infty$ & Re & $D_{0}$ & Re & Do & Re & $\infty$ & Re & Do & $R_{0}$ & $D_{0}$ & Ro & Do & Re & $D_{0}$ & Re & Do & Re & $D_{0}$ \\
\hline Si & La & si & La & si & La & si & La & si & La & si & La & $\mathbf{s i}$ & La & si & La & Si & La & s & La & si & La \\
\hline Do & $\mathbf{s i}$ & Do & 3 & $D_{0}$ & $\mathrm{Si}$ & Do & Si & $D_{0}$ & $\$$ & $\infty$ & si & $D_{0}$ & $\mathrm{si}$ & $D_{0}$ & $\mathrm{si}$ & $D_{0}$ & $\mathbf{s i}$ & Do & $\mathbf{s i}$ & $D_{0}$ & $\mathbf{S i}$ \\
\hline La & Sod & La & Sol & La & sol & La & Sol & La & Sol & La & Sol & La & Sol & Lo & Sol & La & Sol & La & Sol & La & \\
\hline$s$ & La & sa & La & s & La & $\mathbf{s}$ & La & $\mathrm{Si}$ & La & si & La & Si & La & si & La & si & Lo & Si & 7 & & La \\
\hline Sol & $\mathrm{Fa}$ & Sol & $\mathrm{Fa}$ & sol & $\mathrm{Fa}$ & Sol & $\mathrm{Fo}$ & Sol & Pa & Sol & $\mathrm{Fa}$ & Sol & $\mathrm{Fa}$ & Sol & $\mathrm{Fa}$ & Sol & $\mathrm{Fa}$ & Sol & $\mathrm{Fa}$ & Sol & $\mathrm{Fa}$ \\
\hline La & Sol & La & Sol & La & Sol & La & Sol & La & sol & La & Sol & La & Sol & La & sol & La & 99 & La & Sol & Lo & Sol \\
\hline $\mathrm{Fa}$ & Mi & $\mathrm{Fa}$ & Mi & $\mathrm{Fa}$ & Mi & $\mathrm{Fa}$ & M & $\mathrm{Fa}$ & Mi & $\mathrm{Fa}$ & M & $\mathrm{Fa}$ & M & $\mathrm{Fa}$ & M & $\mathrm{Fa}$ & AMi & $\mathrm{Prd}^{\prime}$ & Mi & $\mathrm{Fa}$ & Mi \\
\hline sol & $\mathrm{Fe}$ & Sol & $\mathrm{Fa}$ & Sol & pe & So & $\mathrm{Fa}$ & sol & $\mathrm{Fa}$ & sos & $F$ & sof & $F$ & Sol & $F_{4}$ & Sol & $\mathrm{Fa}$ & sol & $\mathrm{Fa}$ & Sol & $\mathrm{Fa}$ \\
\hline$M_{i}$ & Re & Mi & Re & M & Re & Mi & & & Re & $\mathrm{Mi}$ & Re & & & & & 1 & Re & Mi & Re & M & Re \\
\hline $\mathrm{Fa}$ & Mi & $\mathrm{Fa}$ & $M$ & $\mathrm{Fa}$ & $\mathbf{M}$ & $\mathrm{Fa}$ & & $\mathrm{Fa}$ & Mi & $\mathrm{Fa}$ & & $\mathrm{Fa}$ & & & & $\mathrm{Fa}$ & Mi & $\mathrm{Fa}$ & $\mathbf{M}$ & $\mathrm{Fa}$ & M \\
\hline$R_{e}$ & $D_{0}$ & $R_{\theta}$ & Do & $R_{0}$ & Do & $\sqrt{ }$ & $\infty$ & $R_{e}$ & Do & Re & & Re & & $\omega$ & & Re & $D_{0}$ & Re & $D_{0}$ & $\mathrm{Re}_{\boldsymbol{e}}$ & Do \\
\hline
\end{tabular}

Figure 10: Basic model. 
The diatonic notes 'do, re, mi, fa, sol, la, si' have been associated with quadrant $\mathrm{n} .1$ of each basic element. The notes of the diatonic scale following the notes associated with quadrant n.1 (Fig. 10) have been assigned to the quadrants n. 2, 3 and 4. The column of elements so defined has been repeated along the $\mathrm{x}$-axis to fill the whole $\mathrm{f}(\mathrm{x})$ (Fig. 10).

Following the rules of the method explained in the previous paragraph, G. Donati wrote the following compositions with his usual musical creativity and imagination:

- A rhythmical sequence (score in Fig. 11).

- A melody line (score in Fig. 12).

- A melody merged with the rhythmical base (score in Fig. 13).

- A chord chorale composition 'Courbe donnée' (score in Fig. 14): the title was chosen with the French composition 'Chant donné' in mind; in this case the theme is $\mathrm{f}(\mathrm{x})$.

The application of the method to the composition in Fig. 14 will be analyzed below in detail, focusing particularly on aspects related to the constraints of rules and the degree of freedom left to the composer.

The initial part of $\mathrm{f}(\mathrm{x})$, from $\mathrm{x}=0$ on, and the related first associated basic element have been considered (Fig. 15).

The first step is to define the initial conditions: the composer has to select the first chord and he is quite free to do that. In this example chord A1 in Fig. 16 has been selected by the composer.

This choice has very strong implications for the general atmosphere of the composition, particularly from the point of view of the emotions it generates.

Therefore this choice can or perhaps must create a connection between the sensations aroused by the photograph and those of the music the composer is going to write.

Generally speaking the next chords chosen could be varied in any way, but in this example the composer decided to keep the same typology, a major triad, throughout the composition.

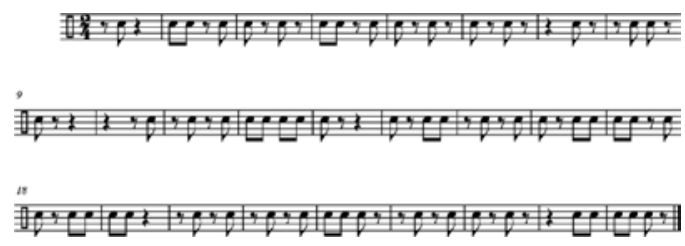

Figure 11: Rhythmical sequence.

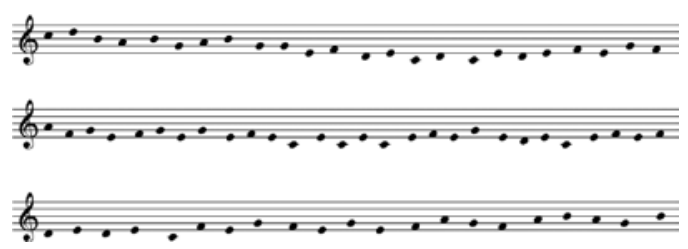

Figure 12: Melody line. 

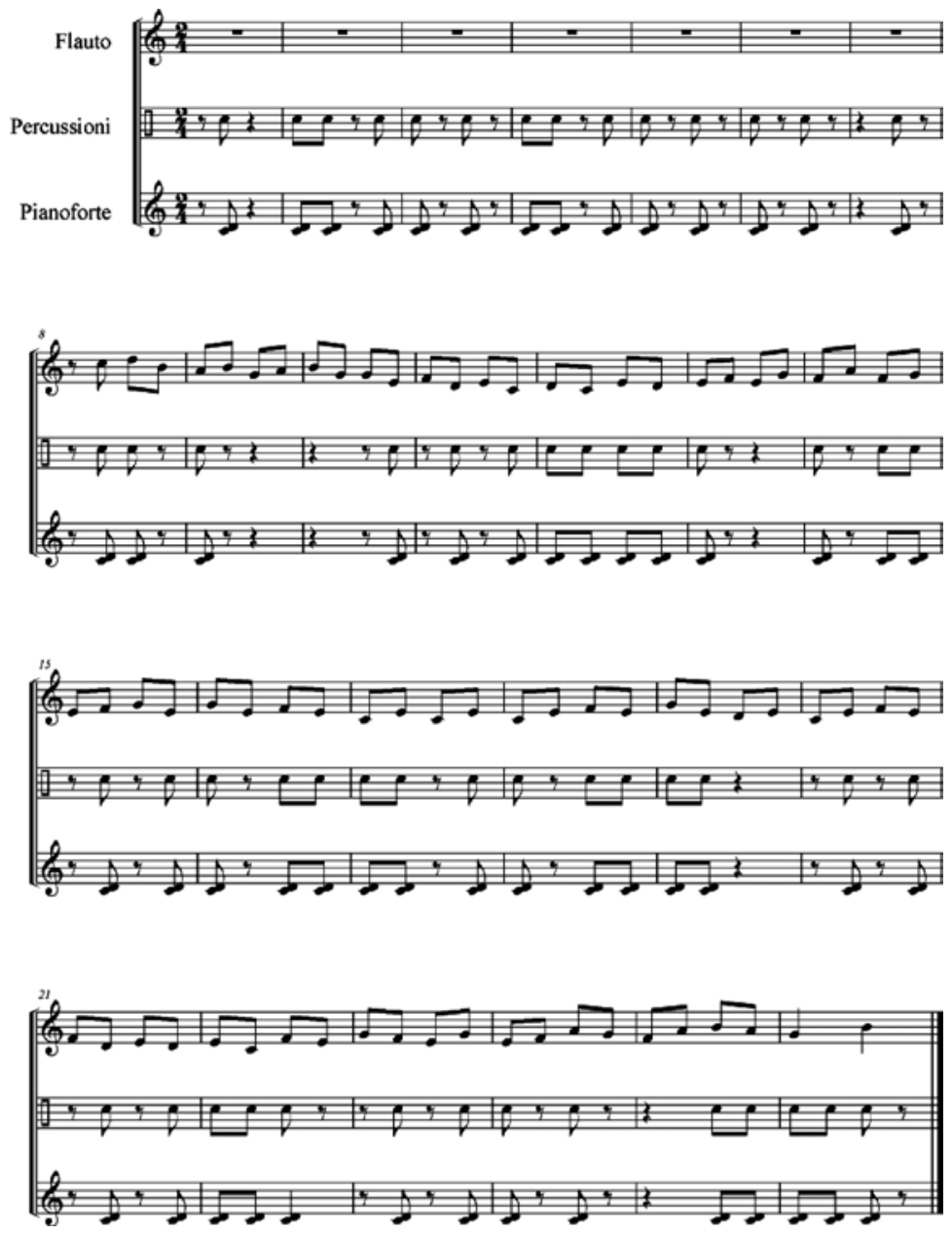

Figure 13: Melody and rhythm.

Figure 16 shows the sequence of chords (A2, A3, A4) generated by starting from A1 in the iterative procedure along the initial part of $f(x)$. The positions of the notes related to the different voices are shown: they are the first chords of the score in Fig. 14.

The main rule guiding the composer in generating the chords is: the note of chord (i) corresponding to the voice in the zone crossed by $f(x)$ is selected as the note at the Bass voice of the chord (i+1).

Synthetically:

$$
\mathrm{B}_{\mathrm{i}+1}=\mathrm{N}_{\mathrm{i}}
$$

$\mathrm{B}_{\mathrm{i}+1}:$ note at the Bass voice of chord (i+1).

$$
\mathrm{N}_{\mathrm{i}}=\mathrm{f}_{\mathrm{i}}\left(\mathrm{V}_{\mathrm{i}+1}\right)
$$

$\mathrm{N}_{\mathrm{i}}$ : note of chord (i); $\mathrm{V}_{\mathrm{i}+1}$ : voice of the zone crossed by $\mathrm{f}(\mathrm{x})$. 

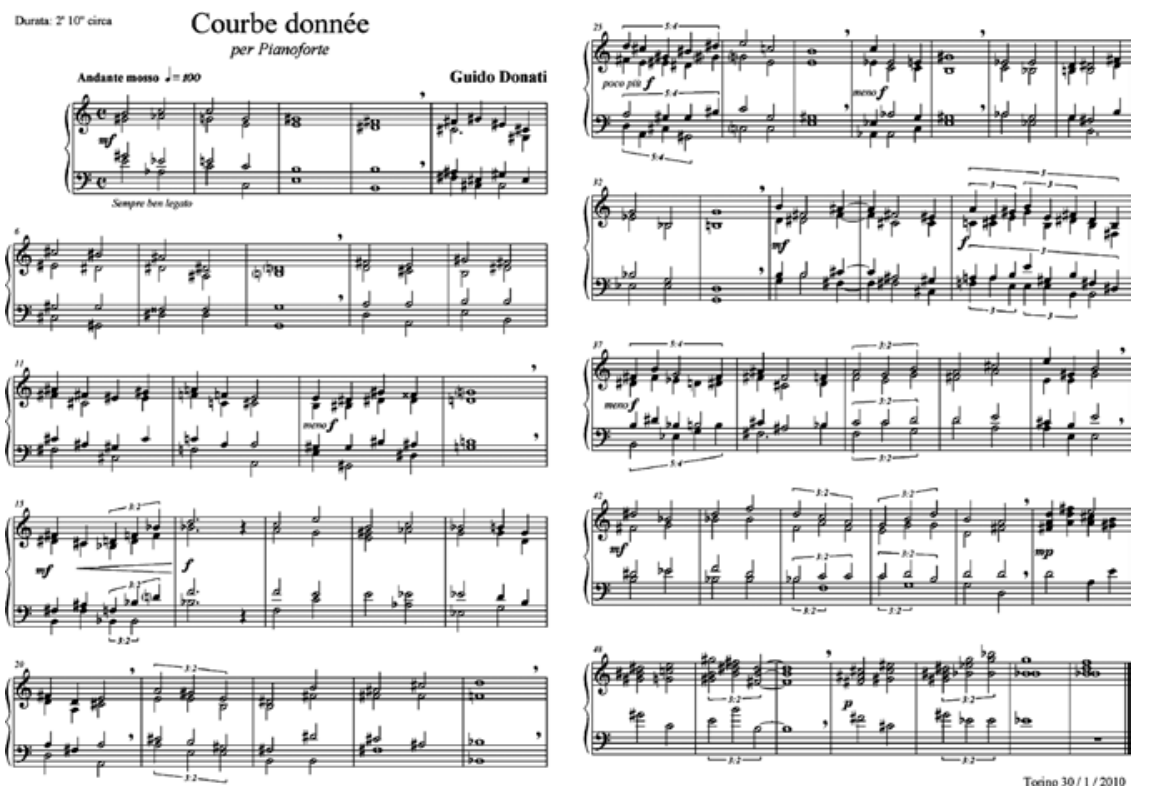

Figure 14: Chorale 'Courbe donnée'.

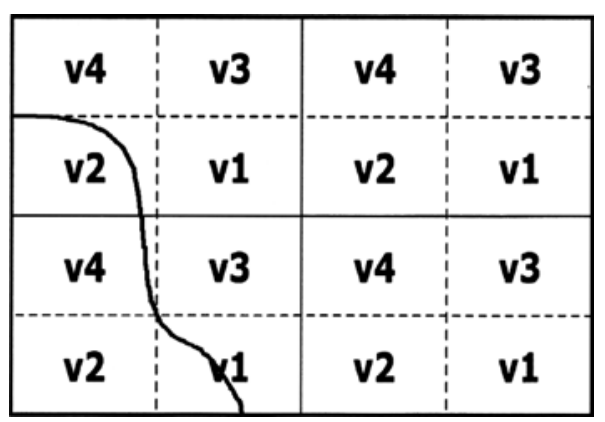

Figure 15: Initial part of $f(x)$.

Now the chords generation procedure will be explained step by step with reference to the part of $\mathrm{f}(\mathrm{x})$ drawn in Fig. 15:

Step 1: $i=1$

The notes of the first chord and their position with reference to the four voices are:

$$
\mathrm{V} 1 \rightarrow \mathrm{mi} \quad \mathrm{V} 2 \rightarrow \mathrm{sol} \# \quad \mathrm{~V} 3 \rightarrow \operatorname{sol} \# \quad \mathrm{~V} 4 \rightarrow \mathrm{si}
$$

Now the chord A2 must be defined: B2, the note at the Bass voice of A2, is 'sol\#', because the $\mathrm{f}(\mathrm{x})$ crosses the zone V2 (Tenor).

At this point the composer must create A2 with 'sol\#' at the Bass voice. But he is completely free to select the other notes of the chords according to the musical ideas he would like to develop. 
Two main decisions must be taken:

- The type of chord (major, minor, seventh, etc.) that is the chord notes.

- The arrangement of the chord notes.

In this example, as mentioned above, the type of chord selected is always the same: major triad.

But the chord notes arrangement is decided by the composer chord by chord with the aim of creating an interesting and beautiful musical composition, taking into account the $f(x)$ path.

In this case, the main possible arrangements are summarized in Fig. 17. The composer selected the first as chord A2.

Step 2: $\mathrm{i}=2$

Now $\mathrm{f}(\mathrm{x})$ goes into V4 (Soprano) zone. Therefore, 'si\#' is selected as the Bass note of chord A3. The composer decided to change 'si\#' enharmonically to 'do'. Then he selected chord A3 from the possible arrangements.

Step 3: $i=3$

Then $\mathrm{f}(\mathrm{x})$ goes into V1 (Bass) zone. Therefore, the chord Bass note does not change, 'do', but the composer decided to define a new chord notes arrangement as in chord A4.

The procedure continues iteratively along the whole $\mathrm{f}(\mathrm{x})$.

Now the rhythmical base is considered (Fig. 11).

\begin{tabular}{|c|c|c|c|c|}
\hline & A1 & A2 & A3 & A4 \\
\hline$v 4$ & Si & Si\# & Do & Sol \\
\hline$v 3$ & Sol\# & Sol\# & Sol & Mi \\
\hline$v 2$ & Sol\# & Re\# & Mi & Do \\
\hline$v 1$ & Mi & Sol\# & Do & Do \\
\hline
\end{tabular}

Figure 16: Chords sequence corresponding to the initial part of $\mathrm{f}(\mathrm{x})$.

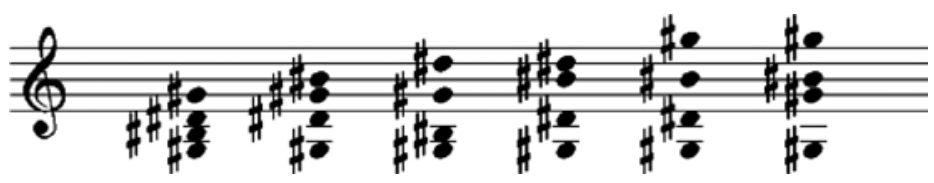

Figure 17: Main arrangements of chord notes. 


\begin{tabular}{|c|c|c|c|c|c|c|c|c|c|c|c|c|c|c|c|c|c|c|c|c|c|}
\hline 4 & 3 & 4 & 3 & 4 & 3 & 4 & 3 & 4 & 3 & 4 & 3 & 4 & 3 & 4 & 3 & 4 & 3 & 4 & 3 & 4 & 3 \\
\hline 2 & 1 & )$^{2}$ & 1 & 2 & 1 & 2 & 1 & 2 & 1 & 2 & 1 & 2 & 1 & 2 & 1 & 2 & 1 & 2 & 1 & 2 & 1 \\
\hline 4 & 3 & $t$ & 3 & 4 & 3 & 4 & 3 & 4 & 3 & 4 & 3 & 4 & 3 & 4 & 3 & 4 & 3 & 4 & 3 & 4 & 3 \\
\hline 2 & 1 & 2 & 1 & 2 & 1 & 2 & 1 & 2 & 1 & 2 & 1 & 2 & 1 & 2 & 1 & 2 & 1 & 2 & 1 & 2 & 1 \\
\hline 4 & 3 & 4 & $\bar{\beta}$ & 4 & 3 & 4 & 3 & 4 & 3 & 4 & 3 & 4 & 3 & 4 & 3 & 4 & 3 & 4 & 3 & 4 & 3 \\
\hline 2 & 1 & 2 & 1 & 2 & 1 & 2 & 1 & 2 & 1 & 2 & 1 & 2 & 1 & 2 & 1 & 2 & 1 & 2 & 1 & 2 & \\
\hline 4 & 3 & 4 & 3 & 4 & 3 & 4 & 3 & 4 & 3 & 4 & 3 & 4 & 3 & 4 & 3 & 4 & 3 & 4 & & & 3 \\
\hline 2 & 1 & 2 & 1 & 2 & 1 & 2 & 1 & 2 & $\sqrt{7}$ & 2 & 1 & 2 & 1 & 2 & 1 & 2 & 1 & 2 & 1 & 2 & 1 \\
\hline 4 & 3 & 4 & 3 & 4 & 3 & 4 & 3 & 4 & 3 & 4 & 3 & 4 & 3 & 4 & 3 & 4 & A & 4 & 3 & 4 & 3 \\
\hline 2 & 1 & 2 & 1 & 2 & 1 & 2 & 1 & 2 & 1 & 2 & 1 & 2 & 1 & 2 & 1 & 2 & & $\mathrm{M}_{2} \mathrm{C}$ & 1 & 2 & 1 \\
\hline 4 & 3 & 4 & 3 & 4 & $\beta$ & 4 & 3 & 4 & 3 & 4 & 3 & $A$ & 3 & 4 & 3 & 4 & 3 & 4 & 3 & 4 & 3 \\
\hline 2 & 1 & 2 & 1 & 2 & 1 & 2 & & 2 & 1 & 2 & 1 & 12 & 1 & & 1 & $\frac{1}{2}$ & 1 & 2 & 1 & 2 & 1 \\
\hline 4 & 3 & 4 & 3 & 4 & 3 & 4 & 3 & 4 & 3 & 4 & 3 & 4 & 3 & & 3 & 4 & 3 & 4 & 3 & 4 & 3 \\
\hline 2 & 1 & 2 & 1 & 2 & 1 & 2 & 1 & 2 & 1 & 2 & $\omega$ & 2 & 1 & 2 & & 2 & 1 & 2 & 1 & 2 & 1 \\
\hline
\end{tabular}

Figure 18: Rhythmical model.

As in the case of the chords sequence, a column of basic elements, each with four quadrants, is defined on the left of the $y$-axis. In this example, a bar with four divisions is considered and their arrangement in the basic element defined as in the chords sequence. Then they are repeated to fill the whole $\mathrm{f}(\mathrm{x})$ as before. But now the bar division numbers are assigned to the quadrants (Fig. 18).

Where the $\mathrm{f}(\mathrm{x})$ crosses the square elements, there a sound impulse is associated to the corresponding bar division number. The other quadrants of the basic elements taken into account, where $f(x)$ does not cross, are associated to pauses: the final result is shown in the sheet in Fig. 11.

Less complex and easier is the definition of the melody line in Fig. 12: notes of the quadrants crossed by $\mathrm{f}(\mathrm{x})$ (Fig. 10) are directly utilized.

Finally the melody and the rhythmical base can be combined (Fig. 13).

\section{CONCLUSIONS}

A general method suitable to guide a composer in writing music based on images has been presented.

An example of an application of the method has been presented to give some idea of its main features and potential.

Future research could proceed in several directions. Of these the following appear particularly interesting and promising:

- The implementation of timbre effects.

- The consideration of images with many $\mathrm{f}(\mathrm{x})$ : which analysis criteria should be taken into account in order to generate the corresponding musical compositions?

- The analysis of the $\mathrm{f}(\mathrm{x})$ as any signal whatsoever by standard techniques, for example, Fast Fourier Transform: what are the implications and the possible developments from the musical point of view?

\section{ACKNOWLEDGEMENT}

Many thanks to Mr. Rocco Minniti, musician and computer expert, who prepared the scores and recorded the compositions. 


\section{REFERENCES}

[1] Schweitzer, A., J.S. Bach, le Musicien-Poète, Parigi, 1905.

[2] Basso, A., Frau Musika, la vita e le opere di J.S. Bach, EDT Musica, Torino, 1981.

[3] Buscaroli, P., Bach, Mondadori, 1985.

[4] Badura-Skoda, P., Interpreting Bach at the Keyboard (trans. A. Clayton), Oxford University Press: USA, 1995.

[5] Donati, G. \& Strona, P.P., Nel Giardino delle Meraviglie, una passeggiata all'ombra delle Variazioni Goldberg di J.S. Bach, Trauben: Torino, 2008.

[6] Berlin, E.A., Ragtime: A Musical and Cultural History, University of California Press, 1984. 